\title{
NAVIGATION AND ELECTRO-OPTIC SENSOR INTEGRATION \\ TECHNOLOGY FOR FUSION OF IMAGERY AND DIGITAL MAPPING PRODUCTS
}

\author{
Alison Brown, NAVSYS Corporation \\ Paul Olson, CECOM
}

\begin{abstract}
$\underline{\text { Abstract }}$
Several military and commercial platforms are currently installing GPS and inertial navigation sensors concurrently with the introduction of high quality visual capabilities and digital mapping/imagery databases. This enables autonomous georegistration of sensor imagery using GPS/inertial position and attitude data. This georegistration capability enables data from digital mapping products to be automatically overlayed on the sensor imagery. In this paper, we describe the system architecture for a Navigation/Electro-Optic Sensor Integration Technology (NEOSIT) software application. The design is highly modular and based on COTS tools to facilitate integration with sensors, navigation and digital data sources already installed on different host platforms.

\section{$\underline{\text { Acknowledgement }}$}

The NEOSIT application is being developed under contract to CECOM (Contract No:

DAAb07-99-C-K755).

$\underline{\text { Keywords }}$

GPS/inertial

NEOSIT

Imagery georegistration 


\section{INTRODUCTION}

In this paper, we describe a baseline system architecture for a Navigation/Electro-Optic Sensor Integration Technology (NEOSIT) software application. This application is being designed to optimally integrate navigation data, sensor imagery and image or terrain database to estimate and correct for errors in each data source. The design is highly modular and based on commercial off-the-shelf tools to facilitate integration with a variety of different navigation and electro-optic sensors and with different sources of digital mapping and imagery databases. The modular design is to allow the NEOSIT application to be used with sensors and navigation already installed on different host platforms and with digital mapping and imagery data sources with varying degrees of precision.

Applications for this system exist for airborne video surveillance where fusion of the navigation, electro-optic and digital mapping data is required to increase the accuracy and timeliness of target coordinates. The driving requirement for airborne video surveillance systems today is to increase the accuracy of target coordinates extracted from the video data and to decrease the time required for processing imagery. To process target coordinates today, from systems such as the Apache Helicopter or Predator UAV (see

Figure 1 and Figure 2), the video data must be georegistered using features selected from within the video images which are at known coordinates, defined by NIMA's Digital Precision Point Database (DPPD) or another equivalent source. This is currently time intensive, requires significant manual operation and is also limited to operation on images where sufficient features are in view for georegistration to occur. With the NEOSIT 
application, the manual intervention is significantly simplified through the data fusion and automated processing techniques employed.

\section{NEOSIT SYSTEM COMPONENTS}

The NEOSIT application is designed to interface with the following components, as illustrated in Figure 3.

\section{$\underline{\text { GPS/Inertial Navigation System }}$}

The NEOSIT uses integrated GPS/inertial navigation data from the host platform to provide the geospatial reference data. Integrated GPS/inertial navigation systems are the core reference for most military aircraft today. More recently, miniaturized, man-portable systems are also being developed that integrate GPS with micro-electro-mechanical (MEM) inertial instrument technology to provide the same integrated navigation capability. Such systems are being developed by the Navy for use in precision targeting (see Figure 4) and are being considered by the Army for applications such as the Land Warrior program.

\section{$\underline{\text { Electro-Optic Sensor }}$}

The NEOSIT application can be used to process data from a variety of different sensors including optical, infra-red (IR) or hyperspectral devices. These sensors must only be capable of providing digital data in a standard image format to the NEOSIT application. The NEOSIT software integrates this information with the navigation and GIS geospatial data to precisely georegister the sensor data against the GPS reference frame. Our baseline design is integrated with a commercial video camera to provide a cost-effective COTS solution. 


\section{GIS Geospatial Data}

The NEOSIT application can accept data from a variety of different digital data sources including government and commercial. This includes rectified imagery (such as the Controlled Image Base (CIB), Digital Precision Point Data Base (DPPDB), digital terrain elevation data (DTED) and vector maps (such as VMAP or commercial equivalents). We achieve this by leveraging a COTS Geographic Information System (GIS) application to support the manipulation of the various types of geospatial data to be fused including maps, terrain models and imagery. Our baseline design includes ESRI's ArcView product which supports analysis and integration of a wide variety of commercial and military geographic information sources.

The NEOSIT design leverages a variety of off-the-shelf software products to provide a seamless integrated solution and to facilitate integrating with a variety of navigation and electro-optic sensor types. The NEOSIT application is designed to integrate the GPS/ inertial, sensor imagery and GIS data to estimate errors in the inertial solution, calibrate for sensor errors and estimate target locations from the sensor imagery. The NEOSIT solution also includes the capability to calibrate for the offsets between different image and terrain geospatially referenced databases (Table 1). To achieve this, the architecture leverages COTS geographic data processing, image processing and image understanding software tools to develop a real-time system for correlation of on-board platform visual and navigation systems with imagery and terrain databases.

\section{PRINCIPLE OF OPERATION}

Using the NEOSIT approach, the georegistration of video imagery can be significantly streamlined by using GPS/inertial navigation information to estimate the location and 
attitude of the video camera. The principle of operation of the NEOSIT georegistration algorithm is illustrated in Figure 5.

The estimated line-of-sight to any feature in the video image, derived in the navigation (North, East, Down) frame, can be computed by transforming the pixel derived line-ofsight vector in camera axes to the navigation frame using the inertial attitude data.

\section{Equation 1}

$$
\underline{l}^{(C)}=\left[\begin{array}{lll}
p_{x} & p_{y} & f
\end{array}\right] / \sqrt{p_{x}^{2}+p_{y}^{2}+f^{2}}
$$

where $p_{x}$ and $p_{y}$ are the target pixel coordinates derived from the image data, and $f$ is the focal length of the camera (in pixel units). The alignment between the camera frame and the inertial body frame is fixed and is defined by the matrix $\mathrm{C}_{C}{ }^{\mathrm{B}}$. The direction cosine matrix derived from the inertial data to transform from body to navigation frame

coordinates $\left(\mathrm{C}_{\mathrm{B}}{ }^{\mathrm{N}}\right)$ can be used to compute the line-of-sight from the camera location to the target location in navigation frame coordinates.

\section{Equation 2}

$\underline{l}^{(N)}=C_{B}^{N} C_{C}^{B} \underline{l}^{(C)}$

Since the camera location is known $\left(\underline{\mathrm{x}}_{\mathrm{c}}\right)$, the target coordinates can be calculated through a least squares solution from multiple image data. The observed line-of-sight to the target provides a measure of the offset between the estimated target location and the observed target location through the following equation.

\section{Equation 3}

$$
\begin{aligned}
& \underline{x}_{T}{ }^{(N)}=\underline{x}_{C}{ }^{(N)}+R \underline{l}^{(N)} \quad R=\left|\underline{x}_{T}-\underline{x}_{C}\right| \\
& \underline{z}=\underline{\hat{x}}_{T}-\underline{x}_{T}=\underline{\hat{x}}_{T}-\underline{x}_{C}{ }^{(N)}-R \underline{l}^{(N)}
\end{aligned}
$$


This residual provides a measure of the following error sources which can be corrected in the NEOSIT integration algorithm to allow fusion of the sensor, navigation and geographic data.

- Error in the initial estimate of the feature coordinates $\left(\underline{\hat{x}}_{T}\right)-$ this corresponds to errors in the GIS data source

- Error in the estimate of the camera location $\left(\underline{\hat{x}}_{C}\right)$ - this corresponds to errors in the navigation solution

- Error in the estimate of the camera attitude $\left(\mathrm{C}_{B}{ }^{\mathrm{N}}\right)$ - this corresponds to errors in the inertial alignment and misalignment angle errors between the inertial system and the video camera

\section{GIS DATA MANAGEMENT}

The GIS data management function in the NEOSIT application is performed using the ArcView® software product provided by ESRI. This allows both raster and vector GIS data sources to be read in a variety of different formats including those listed in Table 2. The following GIS functions are performed by the NEOSIT application.

\section{$\underline{\text { GIS Data Management }}$}

This function manages the storage and retrieval of the GIS data relevant to the particular mission. This is coordinated with the current region of interest using the navigation coordinates provided from the NEOSIT navigation sensor. Retrieval and management of the different data sources is accomplished using the Avenue ${ }^{\mathrm{TM}}$ programming language. 


\section{$\underline{\text { GIS Feature Selection }}$}

The GIS feature selection algorithm operates using a GIS query to select the identified types of features from the GIS database that fall within the field of view of each image. This information is used by the image processing function to narrow the search focus and analysis to be performed and to optimize the algorithm applied for the type of feature being identified. These features can include easily identifiable objects such as signage, road intersections, bridges, or background features such as mountains or buildings. Using the

DLL interface, the GIS feature selection software can direct ArcView ${ }^{\circledR}$ to load and display selected features or regions of interest from the image and terrain database files. In Figure 6, data is shown loaded from multiple Digital Ortho Quadrant files.

\section{$\underline{\text { GIS Data Visualization }}$}

To facilitate correspondence matching between the sensor data and the digital mapping data, the NEOSIT application uses the navigation sensor position and attitude information to map the $2 \mathrm{D}$ display into a $3 \mathrm{D}$ perspective view image. The corresponding $3 \mathrm{D}$ view for a typical sensor location is shown in Figure 7 mapped from the 2D data shown in Figure 6.

\section{IMAGE PROCESSING}

The core function to be performed by the image processing software is to observe the pixel coordinate offsets between the predicted location of a feature in the image and the actual location of the same feature. This observation enables either the navigation errors to be identified from known feature locations or, in the reverse application, to use the pixel offset to identify errors in the recorded location for that feature. 
The basic steps to be followed for generating this observation are illustrated in Figure 8. First a Model is generated of the feature to be matched. Using the a-priori information on the sensor field of view the correct aspect angle and pose of this model is created. The predicted locations of the feature to be matched are also used to Extract the region of interest from the sensor imagery to aid the processing function. Finally the extracted data is matched to the model to observe the offset between the two data sources.

The symbolic processing methodology applies equally well to processing and aligning digital mapping data from both vector and raster imagery sources, as illustrated in the following examples.

\section{$\underline{\text { Feature Matching using Vector Data }}$}

The first example illustrates how actual sensor imagery can be automatically aligned with a vector data model. The first step in the process is to generate the model data. The ArcView program was used to generate a view of the road data over the same area covered by the DOQ view. This reference model is shown in Figure 9 generated from TIGER 96 data files. A region of interest (ROI) is selected to identify the road segment to be used for the matching process. The ROI is shown as the blue area in Figure 9 and is converted by the NEOSIT application to a template for cross correlation with the sensor image. For this example overhead sensor imagery was used to demonstrate the alignment process between the two data sources.

In Figure 10 the DOQ input image is shown with a ROI selected that includes the template segment. This ROI is a larger area to account for possible data errors between the reference model data source and the sensor imagery. The image data is digitally enhanced 
to highlight the road segments which will be used for cross-correlation. The filtered image data is shown in Figure 11.

The NEOSIT matching function is used to search for the template ROI over the image ROI. In Figure 12 the location of the template match with the image data is shown. In this case, the image processing showed that there was a 4 pixel or roughly 12 meter offset between the two data sets at these coordinates.

This example has shown how the NEOSIT matching function can automatically match road segments from a digital vector data source to a digital sensor image of the same area. A similar process can be employed using raster data mapped to a 3-D perspective as shown in Figure 7 to extract the model used for template matching in place of the vector data model generated in Figure 9.

\section{$\underline{\text { Feature Matching Using DEM Data }}$}

In this example, digital elevation model (DEM) data provided by the USGS for the Palmer Lake area was used to generate a 3-D perspective view from the location of an image provided by NAVSYS' GI-Eye GPS/inertial/video system (Brown, Zhang and Reynolds, 1999).

The first step in this process is to extract a skyline profile from the DEM data to be used as the reference model for template matching on the GI-Eye image. This is mapped to the same perspective as the image using the GI-Eye position and attitude data. The reference model generated from this data is shown in Figure 13 overlayed on the GI-Eye image data. The next step in this process is to extract a symbolic representation of the image to be used in the NEOSIT matching process. The results of this step are plotted on Figure 13 in 
yellow. As can be seen, there is a significant offset in the horizontal direction between the model and the image.

The NEOSIT matching algorithm was used to observe the pixel offset between the model data and the image. This algorithm observed an offset of $(48,0)$ pixels between the two data sources. In Figure 14 the Model data corrected for this offset is shown overlaid on the segmented image data.

\section{NEOSIT MODES}

The NEOSIT software has two modes of operation for applying the pixel offset observed from the Image Processing function. Both use optimal estimation techniques to observe system error components. The first mode (GIS Mode) is illustrated in Figure 15. In this mode, the navigation solution is used to provide the geospatial reference frame and the pixel offsets are used to compute the location error of GIS or "target" features extracted from the sensor data. This data is used to build a database which can correct for the baselocation errors in the GIS data source to improve the accuracy of the digital map products. With sufficient data, the digital map errors shown in Table 1 can be reduced to GPS accuracy levels.

The second software mode is designed to enable the navigation sensor errors to be updated, for example during periods where the GPS signals may be denied. In this mode, the processing steps are illustrated in Figure 16. The pixel offsets from features extracted from the sensor data are used to estimate the errors in the onboard navigation solution relative to the georegistered coordinates of these points from the corrected digital mapping data. 
Again, optimal estimation techniques are used to estimate the different error coordinates from the pixel offset data output by the Image Processing function.

\section{CONCLUSION}

The NEOSIT application is being developed under a Small Business Innovative Research (SBIR) contract award for CECOM, Ft Monmouth, New Jersey. Under the first phase of this program, the NEOSIT system was prototyped using NAVSYS' GPS/inertial video targeting system, the GI-Eye (see Figure 17). Under the next phase of this program, the NEOSIT will be developed for use as a generic integrating application for fusing navigation, electro-optic sensor and digital mapping product data. This system will be designed for automated operation to enable real-time correction of digital map coordinates and to provide a backup navigation solution in the event of GPS denial. 


\section{REFERENCES}

Brown, A. K., Zhang, G., Reynolds, D. (1999) Precision Targeting Using GPS InertialAided Sensors, ION 55 ${ }^{\text {th }}$ Annual Meeting, Cambridge, MA;

http://www.navsys.com/9906003.pdf 


\section{TABLES}

Table 1 NIMA Mapping Product Summary ${ }^{1}$

\begin{tabular}{|l|l|l|l|l|}
\hline & CIB & DTED Level 1 & VMAP Level 2 & Digital PPDB \\
\hline Output Unit & 1 degree cell & 1 degree cell & 15 ' tile & 1 degree ZDR \\
\hline Scale & $1: 75 \mathrm{~K}$ & $1: 250 \mathrm{~K}$ & $1: 50 \mathrm{~K}-1: 100 \mathrm{~K}$ & $1: 110 \mathrm{~K}$ \\
\hline Digital Storage & $13 \mathrm{~GB}$ & $2.9 \mathrm{MB}$ & varies & $26 \mathrm{~GB}$ \\
\hline Horiz Accuracy & $<61 \mathrm{~m}$ & $50 \mathrm{~m}$ & $50-200 \mathrm{~m}$ & Classified \\
\hline Vert Accuracy & N/A & $30 \mathrm{~m}$ & $0.5-2$ contours & Classified \\
\hline
\end{tabular}

Table 2 NEOSIT GIS Data Sources

\begin{tabular}{|l|l|}
\hline Military Products & Commercial Products \\
\hline Controlled Image Base TM (CIB TM) & Digital Orthophoto Quadrangles (DOQ) \\
Digital Terrain Elevation Data (DTED) & Digital Elevation Model (DEM) \\
Vector Smart Map Product (VMAP) & Digital Raster Graphics \\
& TIGERß 95 \\
\hline
\end{tabular}

1 "Mapping, Charting \& Geodesy Handbook", Naval Aviation Systems Team, AVDEP-HDBK-12,

Version 2 (http://www.fas.org/man/dod-101/navy/docs/pc_hndbk.html) 


\section{FIGURES}

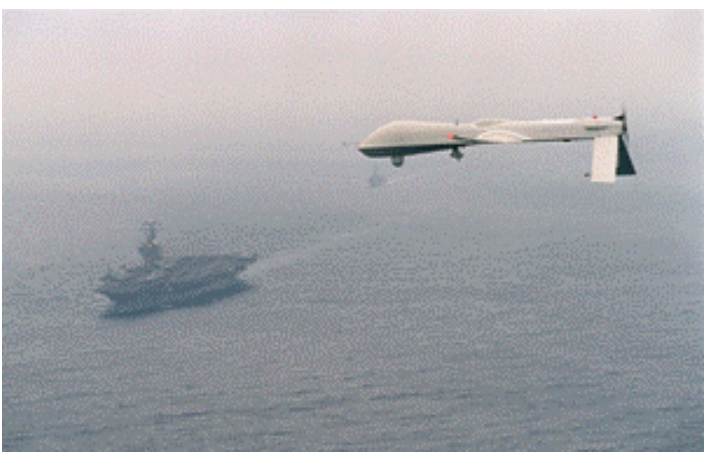

Figure 1 Predator UAV

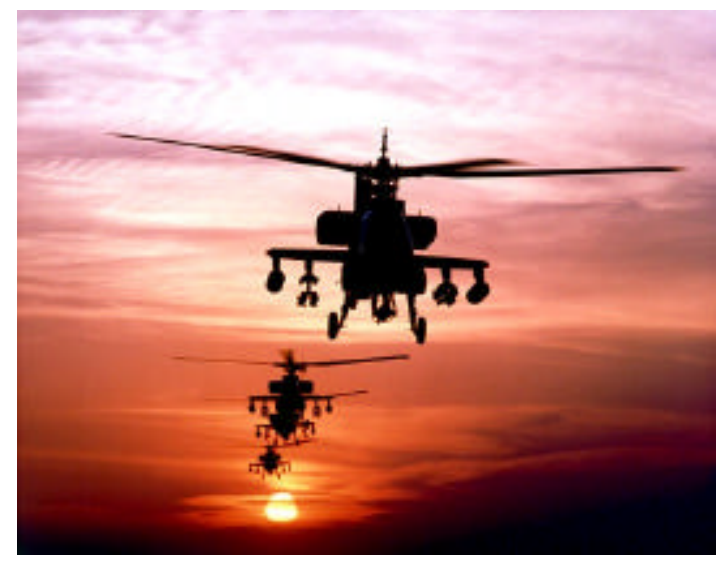

Figure 2 Apache Helicopter

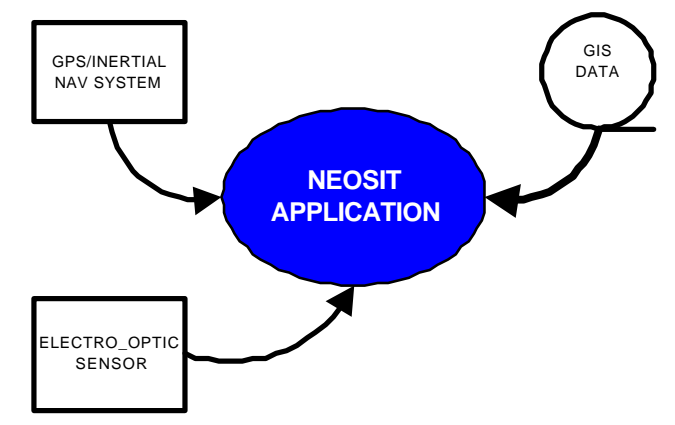

Figure 3 NEOSIT Component Interfaces 


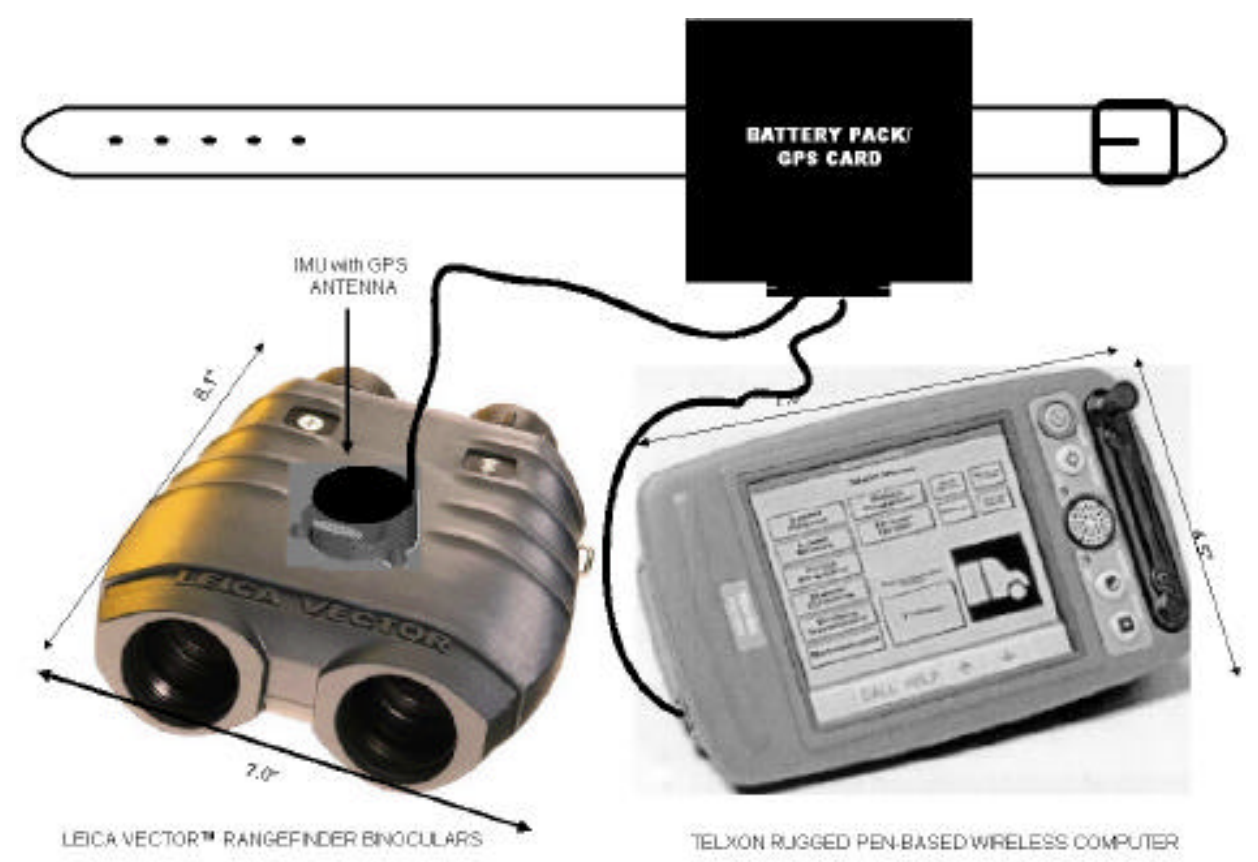

Figure 4 SPOTS Man-portable GPS/IMU Targeting Sensor

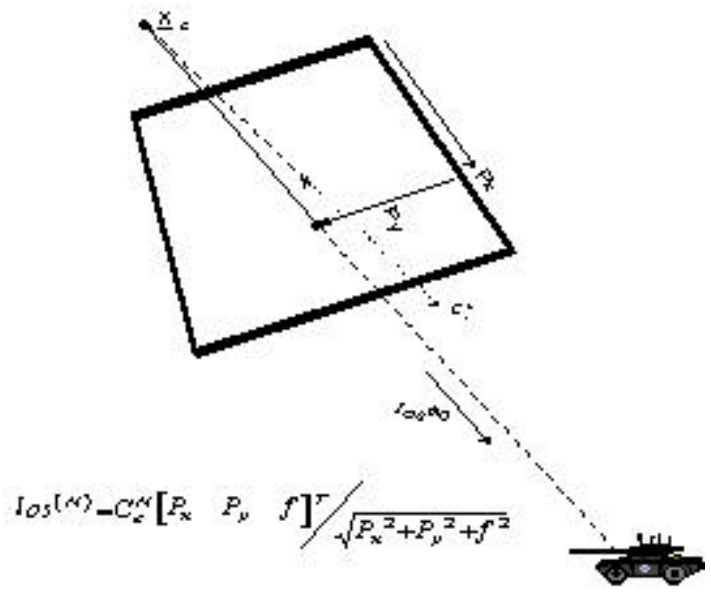

Figure 5 GPS/Inertial Georegistration 


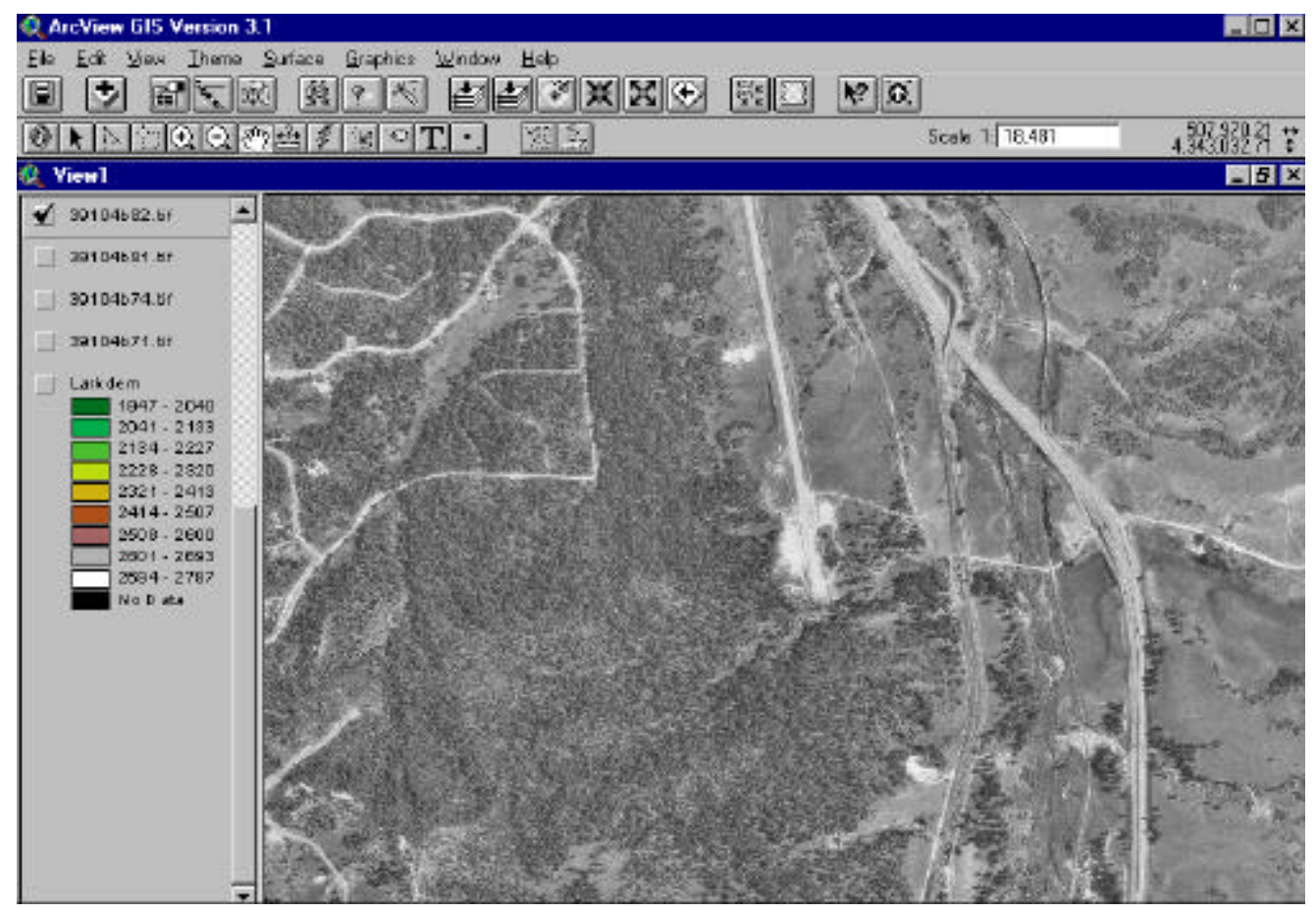

Figure 6 ArcView 2D view window with 5 themes loaded

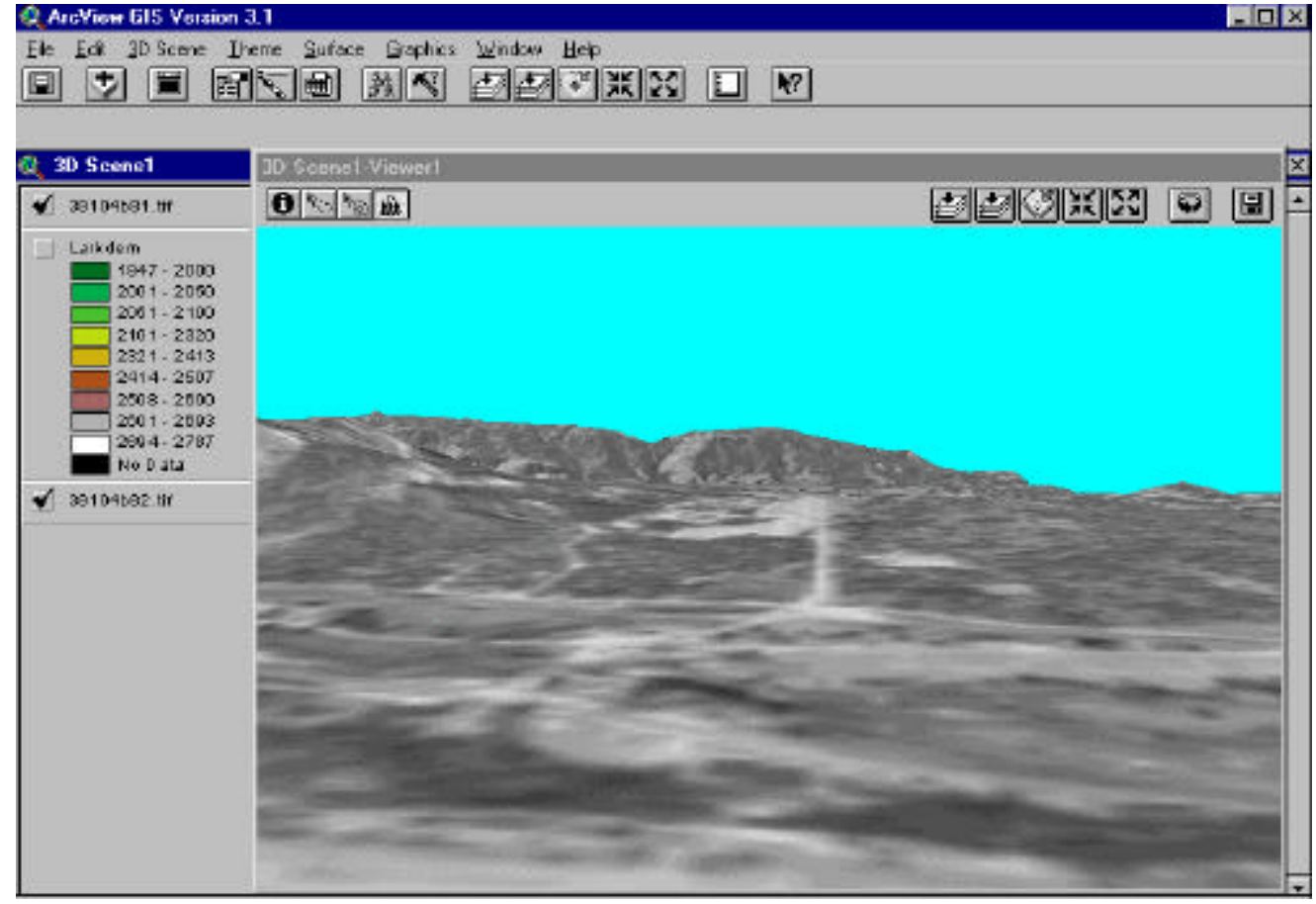

Figure 7 ArcView 3D view window looking west from Larkspur I-25 interchange 


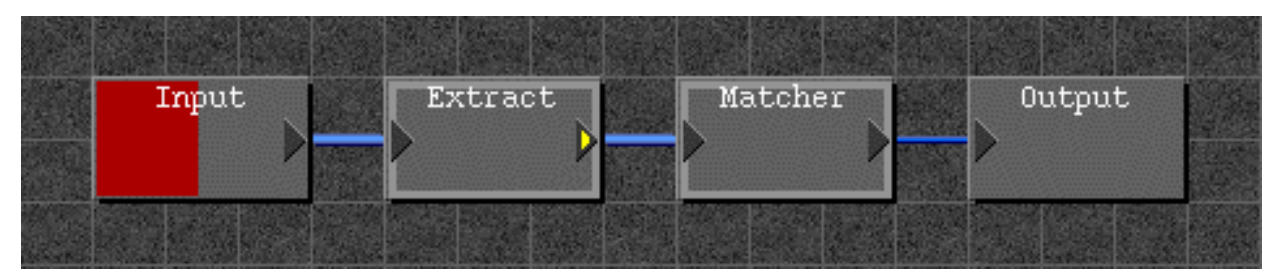

Figure 8 Symbolic Processing Methodology

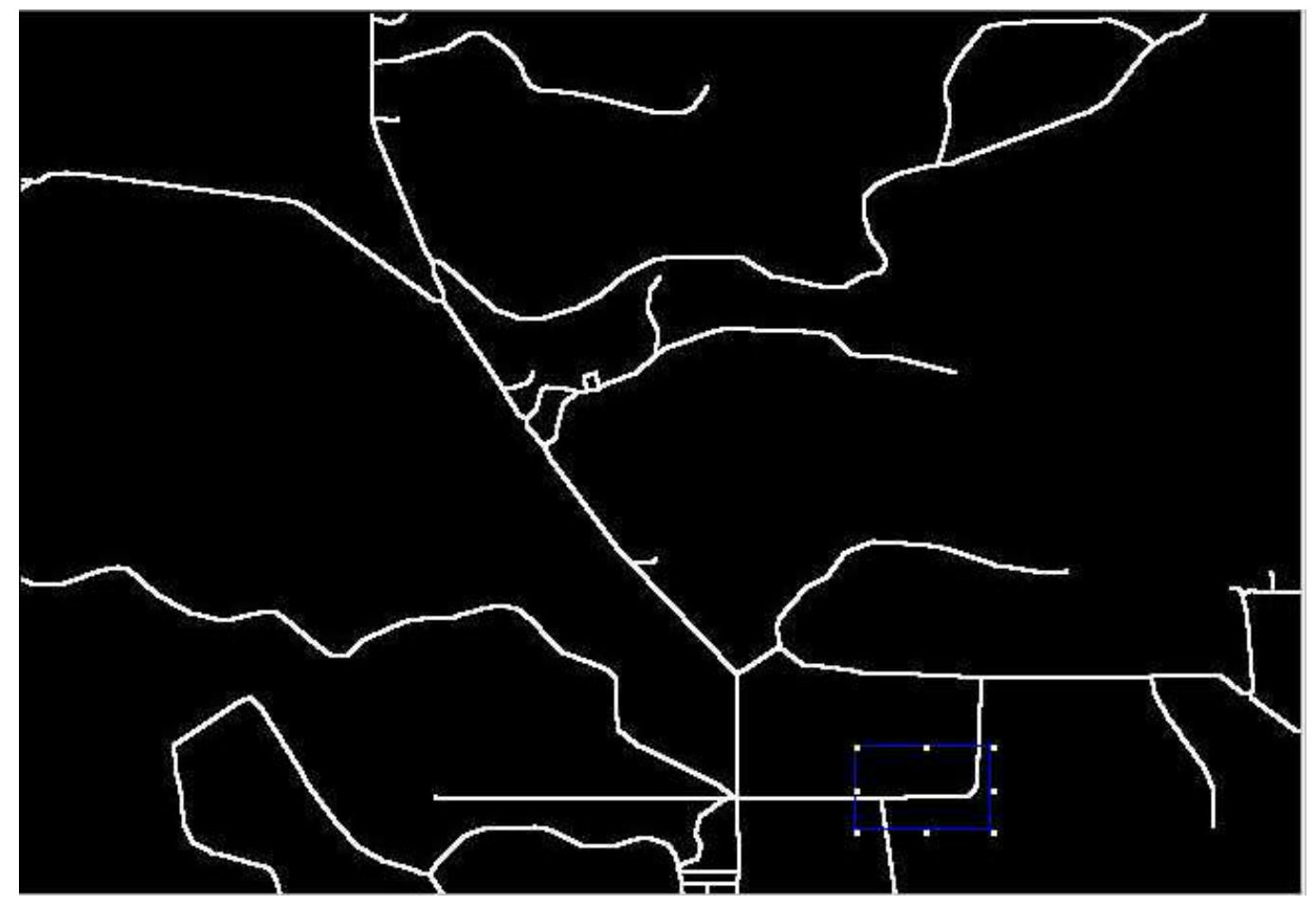

Figure 9 Reference Model with feature selected 


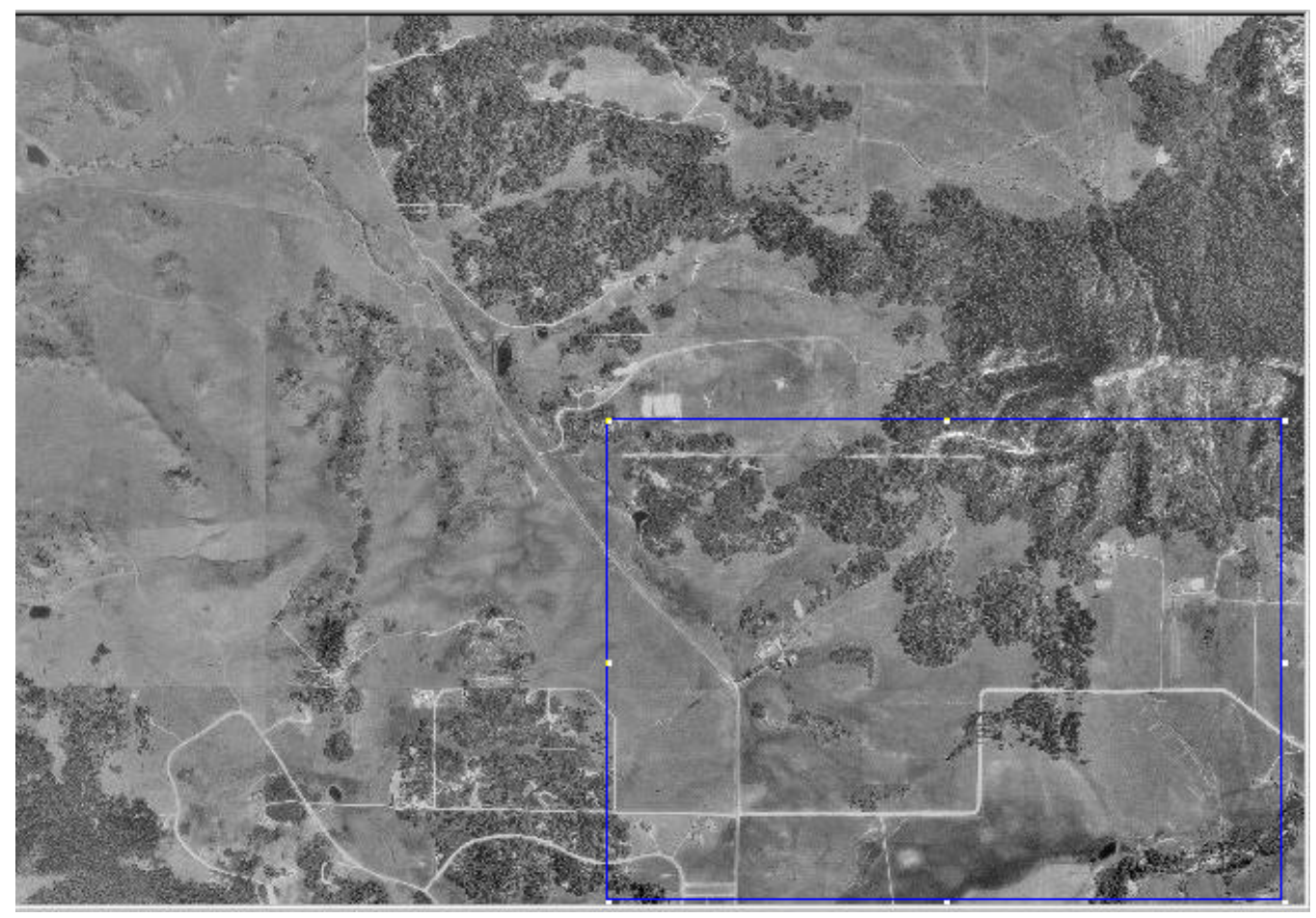

Figure 10 Input Image with area to search selected

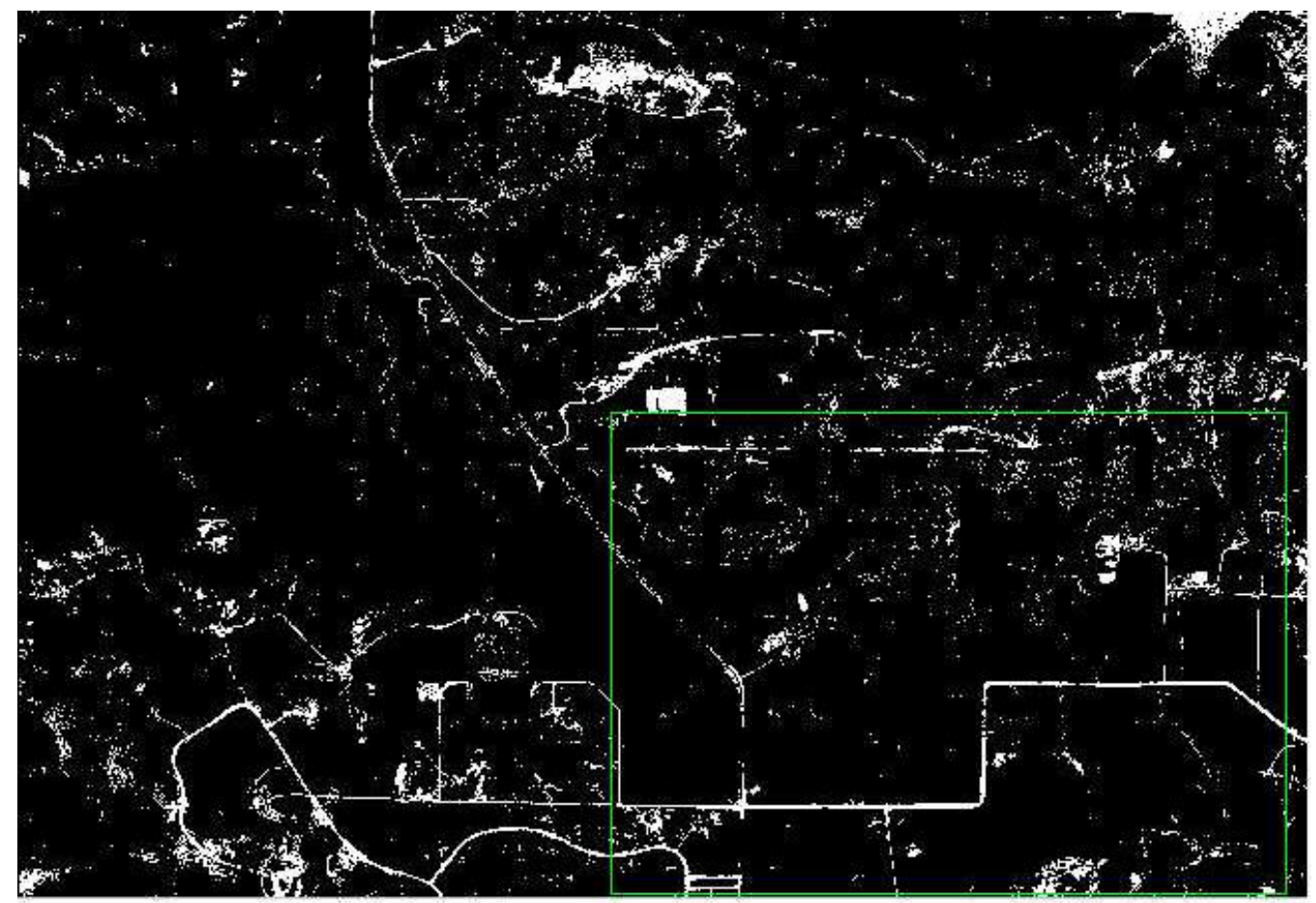

Figure 11 Input image after filtering to bring out roads 


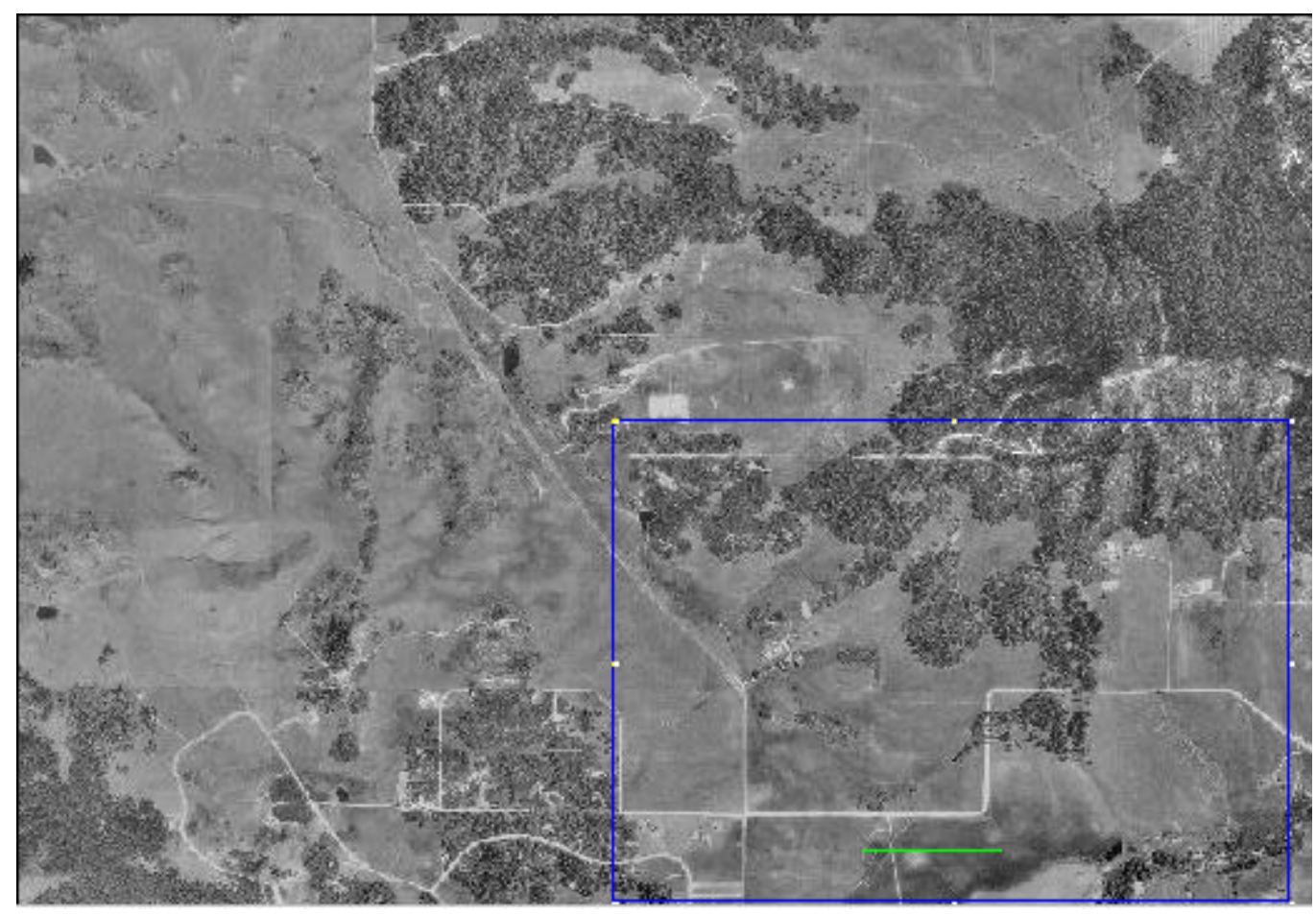

Figure 12 Input image with feature found within the search region using crosscorrelation

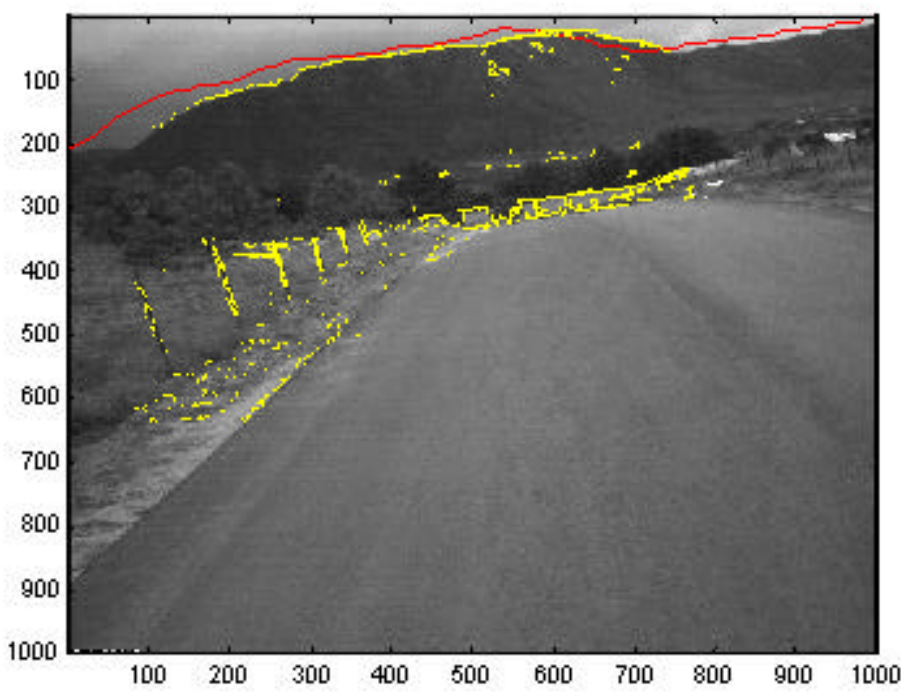

Figure 13 GI-Eye data with DEM model superimposed 


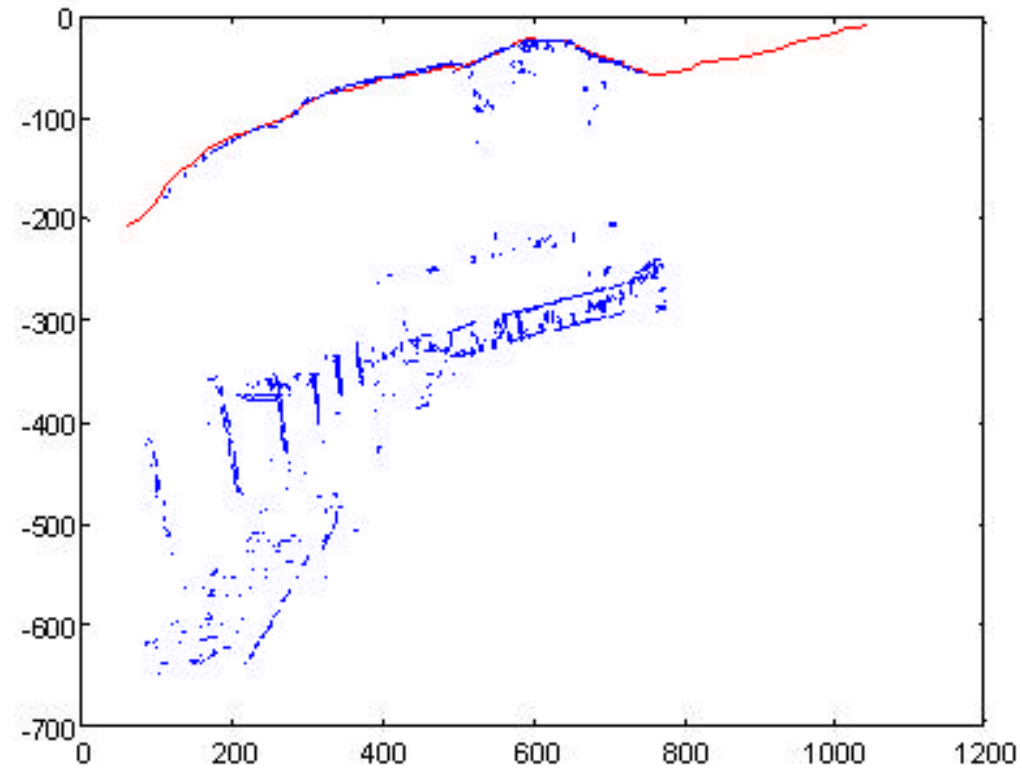

Figure 14 Symbolic image representation and Matched model

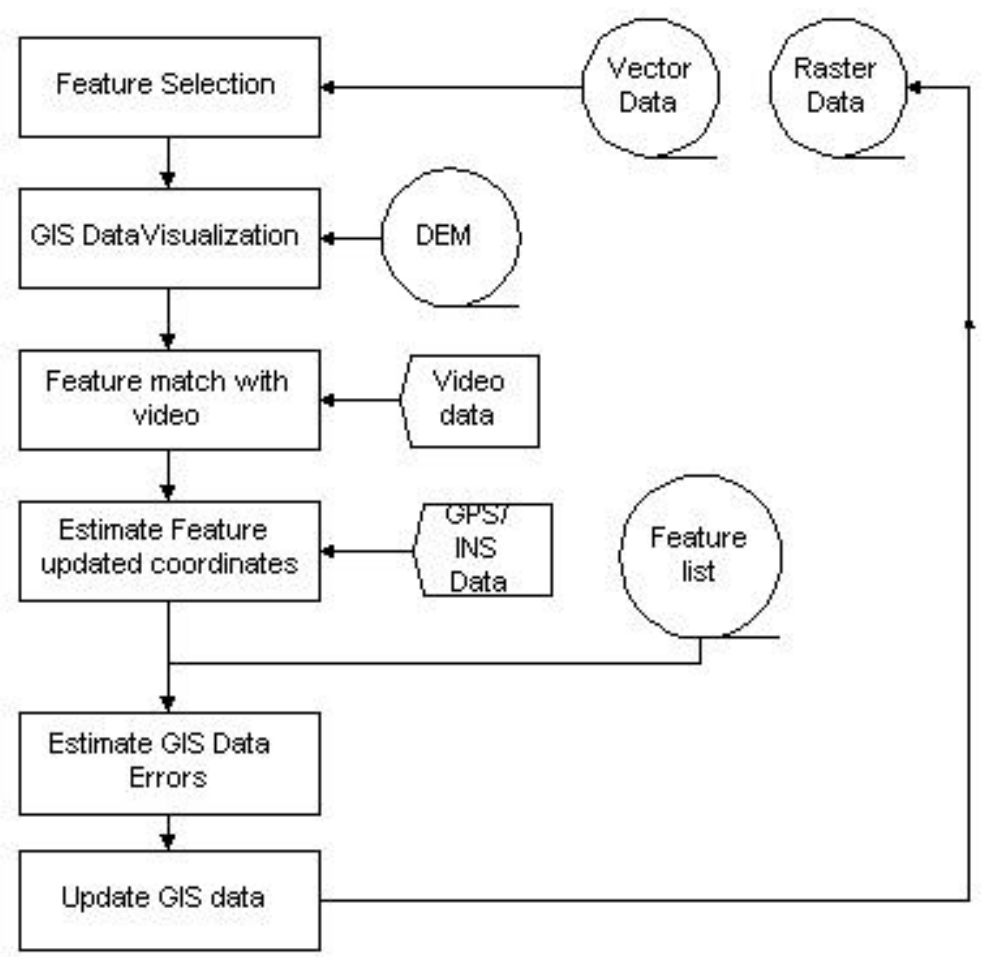

Figure 15 GIS Update Software Mode 


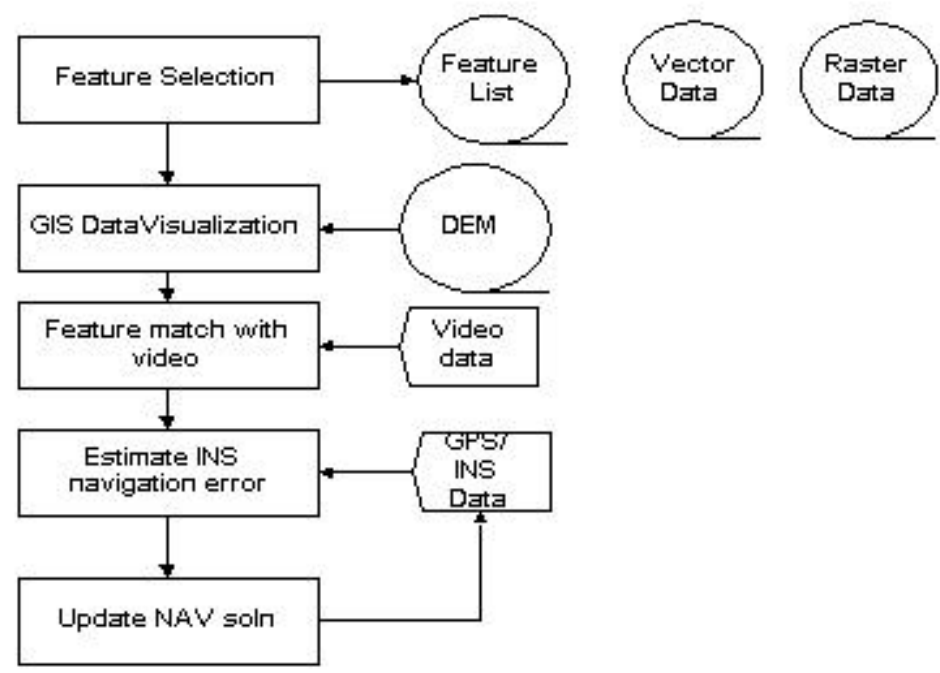

Figure 16 Navigation Update Software Mode

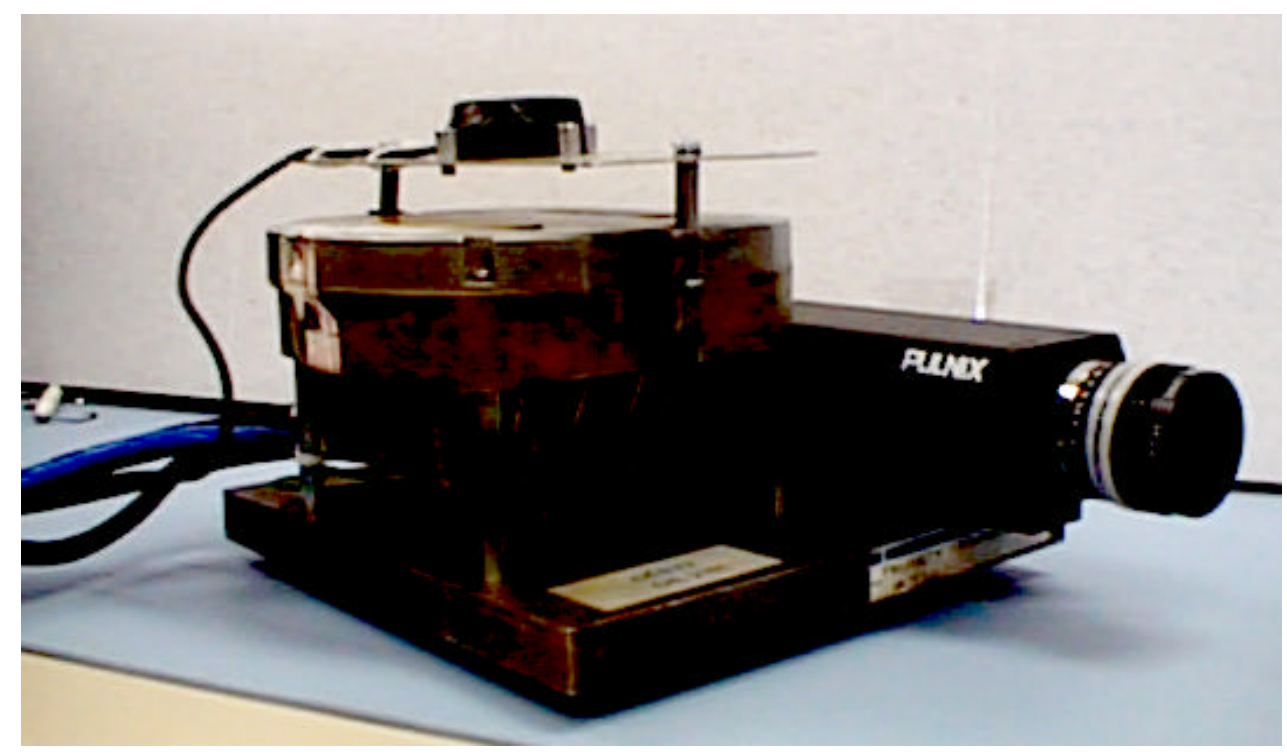

Figure 17 GI-Eye GPS/Inertial/Video Targeting System 